\title{
Einsatz und Nutzung von Social Software in kooperativen Forschungsprojekten
}

\author{
Christoph Lattemann, Miriam Kallischnigg, Stefan Stieglitz. \\ Corporate Governance und E-Commerce, \\ Universität Potsdam
}

\section{Einleitung}

Social Softwaretechnologien und -verfahren wie Weblogs, Wikis und Soziale Netzwerke bilden neue Möglichkeiten zum Informations- und Wissensaustausch und zur Zusammenarbeit in und zwischen Organisationen (McAfee 2009, Stieglitz 2008). Obgleich einige dieser technologischen Ansätze nicht völlig neu sind, hat der Trend des Web 2.0 den Begriff der Social Software mit erfasst und popularisiert. O’Reilly beschreibt Web 2.0 als Plattform, die auf einer Architektur der Beteiligung basiert, die Services im Paket anbietet, auf der Datenquellen vermischbar sind und dadurch Datentransformationen möglich werden (O'Reilly 2005, Szugat et al. 2006). Social Software stellt heute wesentliche Inhalte dessen dar, was Web 2.0 ausmacht und fasst die „Webanwendungen zusammen, die dazu dienen, die Kommunikation innerhalb menschlicher Netzwerke zu unterstützen."(Szugat et al. 2006, S. 13). Allen (2004) vereint all solche Anwendungen unter dem Begriff Social Software, die die Interaktion innerhalb einer Gruppe stärkt. Damit forciert Social Software Beziehungen, die innerhalb von bereits formierten Gruppen bestehen.

Über die Einsatz- und Nutzungsmöglichkeiten von Web 2.0-Anwendungen wurde in den letzten Jahren ausführlich diskutiert (z. B. Bartel 2006; Orth 2009). Darüber hinaus existieren eine Reihe von Studien, wie BITKOM 2008, Berlecon Research 2007, Economist Intelligence Unit 2007/2008, McKinsey 2007/2008, Techconsult 2008, die die zunehmende Implementierung von Web 2.0-Technologien in Unternehmen weltweit belegen und die zentralen Erkenntnisse im Einsatz von Social Software in Unternehmen verdeutlichen. Bislang unbeachtet blieben jedoch die Verwendung und Potenziale dieser Technologien in (Forschungs-)Verbundprojekten.

Eine solche vergleichende Analyse der Einsatzmöglichkeiten und Mehrwerte von Social Software für (Forschungs-) Verbundprojekten mit der Nutzung in Unternehmen kann dazu beitragen, Best Practices zu identifizieren und Projektleitern einen Soll-Ist-Vergleich für die eigenen Projekte zu ermöglichen. Um diese bestehende Forschungslücke zu schließen wurde für diesen Beitrag eine Online-Umfrage unter Projektleitern von aktuell laufenden DFG Forschungsverbundprojekten durchgeführt.

Im internationalen Vergleich stehen „Deutschland und Frankreich am Schluss der Skala der Web 2.0Nutzung in Europa.“ (Krause 2009, Geleitwort). Diese Studie kann helfen, aufzuzeigen, ob und inwiefern Forschungsprojekte in Deutschland einen Treiber bzw. einen Motor für die Nutzung von Social Software, speziell in Deutschland darstellen könnten, um in der Anwendung dieser Technologien im internationalen Vergleich aufzuschließen. In wissenschaftlicher Hinsicht stellt diese Untersuchung eine Basis für weitere vertiefende Studien dar und bietet gleichzeitig die Möglichkeit, Best Practices für die Projektarbeit aufzudecken. Die Untersuchungsergebnisse geben gezielt Aufschluss darüber, inwieweit Social Software in Forschungsprojekten Anwendung findet und welche Erfahrungen diesbezüglich gemacht wurden.

Im Folgenden werden Fragestellungen aus der Literatur und bestehenden Analysen zur Nutzung von Social Software abgeleitet und diese anhand der Ergebnisse der Umfrage in Forschungsprojekten validiert. Hieraus wird abschließend ein Fazit aus dem angestellten Vergleich zum Nutzen von Social Software in Projekten und in Unternehmen gezogen.

\section{Einsatz von Social Software in kooperativen Forschungsprojekten}

Studien (vgl. Lattemann et al. 2009) belegen die erfolgreiche Implementierung von Web 2.0-Technologien in Unternehmen und heben die Bedeutung des Einsatzes und der Nutzung von Social Software für die zukünf- 
tige Zusammenarbeit in und zwischen Unternehmen hervor. Die Economist Intelligence Unit aus dem Jahr 2008 prognostiziert hierüber hinaus, dass die Struktur von Projektteams in Zukunft aufbrechen und sich räumlich verteilen wird (Economist Intelligence Unit 2008). Dies lässt erwarten, dass der Bedarf nach einer virtuellen Vernetzung auch in der Projektarbeit weiter wachsen wird.

Social Software, als eine Untermenge von Web 2.0-Technologien und Verfahren, umfasst Dienste, die das menschliche Sozialverhalten unterstützen, erweitern und daraus einen Mehrwert für den Austausch und die Generierung von Wissen erzeugen (Koch 2008a). Verschiedene Web 2.0-Technologien können in Weblogs, Wikis, Diskussionsforen, Social Tagging bzw. Social Bookmarking, Social Networking und Instant Messaging unterschieden werden (Koch und Richter 2007, S. 11-12; Bächle 2006). Es gibt eine Vielzahl von Definitionen von Social Software, die jeweils auf verschiedene Aspekte fokussieren. So werden beispielsweise unter Social Software Softwaresysteme verstanden, „welche die menschliche Kommunikation und Kollaboration unterstützen“ (Bächle 2006, S. 121). Mit Fokus auf den zu schaffenden Mehrwert, werden diese Technologien auch als „software which supports, extends, or derives added value from, human social behavior [...]” verstanden (Coates 2005). Diese Definition reflektiert die Beobachtung, dass Wikis, Blogs und Foren in Unternehmen zunehmend intensiver eingesetzt werden, um Mitarbeiter verstärkt in Unternehmensprozesse einzubinden und um kollaboratives Arbeiten sowie die Entwicklung von Innovationen zu fördern (Döbler 2007, Lattemann et al. 2009). Online Communities dienen im B2B- und B2C-Bereich u.a. der Verfolgung von Customer-Integration-, Open-Innovation- oder Customer-Relationship-Ansätzen (Piller und Stotko 2003; Chesbrough 2003), um den Kontakt zum Endverbraucher bzw. zu anderen Stakeholdern zu intensivieren. In ähnlicher Weise werden zunehmend Web 2.0-Technologien für die unternehmensinterne Kommunikation und Kollaboration herangezogen.

Eine gezielte Integration von Social Software kann zu einem besseren Informations- und Wissensmanagement in Unternehmen führen. Dies trifft insbesondere für räumlich verteilte Arbeit zu, die in Projekten üblicherweise stattfindet (Koch 2008b). Um die Potentiale jedes Einzelnen in einem Team auszuschöpfen, besteht die Notwendigkeit einer Technologie-orientierten Zusammenarbeit (Scheer 2008). Nach DIN 69901 (GPM 2009) ist ein Projekt als ein Vorhaben definiert, das im Wesentlichen durch Einmaligkeit der Bedingungen in ihrer Gesamtheit gekennzeichnet ist, wie z. B. der Zielvorgabe, zeitliche, finanzielle, personelle oder andere Bedingungen sowie einer Abgrenzung gegenüber anderen Vorhaben. In dieser Definition wird implizit die hohe Komplexität, die durch die Projekte gekennzeichnet sind, thematisiert.

Verschiedene Studien (Lattemann et al. 2009) zeigen hierüber hinaus, dass für große, mitarbeiterstarke Unternehmen der Einsatz von Web 2.0 aufgrund einer möglichen Einbindung in das Wissensmanagement besonders interessant ist. Dies begründet sich daraus, dass in diesen Firmen eine stärkere Notwendigkeit besteht, die Vernetzung und Kommunikation zwischen Mitarbeitern und zu Geschäftspartnern, Zulieferern und Kunden technologisch zu unterstützen. Problematisch stellt sich hierbei die Erfolgsmessung eines solchen Netzwerkes dar. So benennen zwar Hagel und Armstrong (2006) vier Parameter zur Erfolgsmessung (Transaktion, Inhalte, Loyalität und Profildaten), geben jedoch keinen Handlungsrahmen zur Operationalisierung und Bewertung der Kriterien vor. Hingegen existieren bereits Modelle, die eine ökonomische Analyse der Kosten und Nutzen von virtuellen Unternehmensnetzwerken ermöglichen (Stieglitz et al. 2008).

Eine Studie von BITKOM (2008) und eine weitere Studie von Berlecon Research (2007) haben identifiziert, dass insbesondere solche Unternehmen Vorreiter in der Nutzung Sozialer Software sind, die über komplexe Strukturen (mit verschiedenen Standorten) und eine große Mitarbeiterzahl (mehr als 5.000 Mitarbeiter) verfügen. Diese nutzen Web 2.0-Technologien intensiver als kleine Unternehmen oder solche mit einem einzigen Standort. Wikis und Blogs werden von 20\% bis 40\% (in Abhängigkeit von der jeweiligen Studie) der großen deutschen Unternehmen eingesetzt, während nur etwa 10\% bis $20 \%$ der kleinen und mittleren Unternehmen diese Plattformen nutzen (Techconsult 2008, BITKOM 2008).

Diese Ausführungen zur Nutzung von Social Software in verteilten Strukturen stützen die Annahme, dass auch innovative Forschungs- bzw. Verbundprojekte, an denen mehrere Partner an verteilten Standorten arbeiten, von einer intensiven Nutzung von Web 2.0-Technologien profitieren können und daher ggf. Best Practices identifiziert werden können.

Die genannten Unternehmensstudien kommen weiterhin zu dem Ergebnis, dass für die interorganisatorische Zusammenarbeit Wikis, Blogs, Foren und Soziale Netzwerke von ca. 30\% bis 35\% (mit steigender Tendenz) der Firmen genutzt werden. Andere Web 2.0-Technologien wie Mash-Ups, Soziale Netzwerke und RSS Feeds werden mit einer deutlich geringeren Intensität in Unternehmen eingesetzt. Diese Ergebnisse führen zu der Annahme, dass die Social Software Werkzeuge, die am häufigsten in Unternehmen genutzt werden, ebenfalls auch in kooperativen Forschungsprojekten verstärkt eingesetzt werden. Hieraus würde folgen, dass auch in kooperativen Forschungsprojekten vor allem Wikis, Blogs und Foren zum Einsatz kommen, während 
andere Typen von Social Software nicht oder nur in geringem Ausmaße genutzt werden. Diese Annahme wird anhand der folgenden Fragestellungen überprüft:

1. Wie intensiv wird Social Software in Forschungsverbundprojekten der Deutschen Forschungsgemeinschaft (DFG) eingesetzt?

2. Welche Typen von Social Software werden maßgeblich in Forschungsverbundprojekten eingesetzt?

3. Inwiefern kann der Einsatz von Social Software in Forschungsverbundprojekten für Unternehmen eine beispielhafte Rolle spielen?

Wird ein intensiverer Einsatzes von Social Software in Forschungsverbundprojekten beobachtet, könnten Unternehmen von Forschungsverbundprojekten lernen. In diesem Fall könnten Projekte der DFG einen Beitrag leisten, Deutschland in der Nutzung von Web 2.0 voranzubringen. Eine geringe Nutzung von Web 2.0-Technologien in Forschungsverbundprojekten der DFG würde andererseits nahelegen, dass Unternehmen verstärkt in solche Projekte eingebunden werden sollten, um durch einen Wissensaustausch und -transfer den Einsatz von Web 2.0 in dieser Domäne zu intensivieren.

\section{Einsatz von Social Software in von der DFG geförderten Forschungsverbundprojekten}

Um die aufgestellten Fragen beantworten zu können, wurde eine Umfrage zur Nutzung von Social Software in Forschungsverbundprojekten durchgeführt, die von der DFG gefördert werden. Die in dieser Studie untersuchten DFG-Forschungsprojekte sind durch eine stark räumlich verteilte Struktur mit Projektpartnern an verschiedenen Standorten geprägt. Es wurde eine Online-Befragung mittels der Open Source-Software Lime Survey durchgeführt. Die Umfrage richtete sich an Projektleiter von ausgewählten DFG-Projekten. Für die Analyse wurden Verbundförder- und -forschungsprogramme aus den Bereichen „Koordinierte Programme“ und „Wissenschaftliche Infrastruktur“ der DFG ausgewählt. Die analysierten Programme gehen über die Förderung einzelner Personen oder die Verleihung von Preisen hinaus und basieren auf verteilten Wissenschaftlerteams. Eine Begrenzung der Studie auf DFG-Projekte wurde aus zwei Gründen vorgenommen. Erstens können über die GEPRIS Datenbank der DFG die Stammdaten der Projekte leicht identifiziert werden. Die Ansprache von Projektleitern wurde vereinfacht. Zweitens kann angenommen werden, dass in DFGProjekten eine State-of-the-Art Forschung betrieben wird, die auch in den Bereich der Projektorganisation und den Softwareeinsatz wirkt.

In der Analyse wurde zwischen Diskussionsforen, Instant Messenger, Wikis, (Web)Blogs, Social Bookmarking sowie Soziale Netzwerke unterschieden, da sämtliche dieser Instrumente das Potenzial besitzen, Kollaboration und verteilte Teamarbeit zu unterstützen.

\subsection{Fragebogen und Auswertung}

Der erstellte Online-Fragebogen gliederte sich in zwei Abschnitte: Der erste Teil behandelte Fragen zur Nutzung von Social Software in den Forschungsprojekten. Der zweite Teil behandelte Eckdaten des zu Grunde liegenden Projekts. Während der erstgenannte Teil dazu diente, den Einsatz ausgewählter Web 2.0Technologien hinsichtlich der Qualität, der Nutzung, der Vorteile und Bedenken zu ermitteln, wurde im Teil 2 eine Einordnung der Projekte hinsichtlich Größe, Komplexität und IT-Nähe erfasst. Die Umfrage wurde anonym durchgeführt. Der Fragebogen enthielt ausschließlich geschlossene Fragen, mit vorgegeben Auswahlmöglichkeiten auf verschiedenen Skalenniveaus: Einfach-, Mehrfachauswahl und Likert-Skalen. Die Auswertung erfolgte mit PAWS 17 und Microsoft Excel.

\subsection{Erhebungsmethodik}

Für die Erhebung der Teilnehmerdaten wurde der Research Explorer GEPRIS (http://gepris.dfg.de/gepris/) der DFG genutzt. Über die Funktion ,Exploration“ konnte die konkrete Auswahl je DFG-Programm für die Zusammenstellung der relevanten Personen für die Umfrage definiert werden. Weithin traten Einschränkungen in der Auswahl der Projekte auf, da teilweise Projekte vom gleichen Projektleiter geführt wurden, oder über die angegebene Email-Adresse kein konkreter Ansprechpartner erreichbar war. 
Die Erhebung der für die Umfrage grundlegenden Datensätze wurde vom 01.08.2009 bis zum 05.08.2009 vorgenommen. Die Online-Umfrage wurde im Zeitraum 03.09.2009 bis 13.09.2009 durchgeführt. Die ausgewählten Projektleiter wurden per Email angeschrieben. Eine Nachfassaktion erfolgte eine Woche nach der ersten Anfrage per Email. 131 von 584 Teilnehmern hatten während des Befragungszeitraums den Fragebogen ausgefüllt (22,43\%); 81 Fragebögen wurden vollständig ausgefüllt (13,87\%)(Tabelle 1). War eine Person für mehrere Projekte als Projektleiter definiert, wurde dieser auch nur einmal angeschrieben. Dieser Projektleiter wurde aufgefordert, den Fragebogen auf ein von ihm frei ausgewähltes Projekt zu beziehen.

Da DFG-Projekte jedoch keine homogene Masse darstellen, sondern im Gegenteil sehr hohe Spezialisierungsgrade in verschiedenen Forschungsfeldern aufweisen, muss dieses bei der Ergebnisinterpretation berücksichtigt werden. So kann angenommen werden, dass beispielsweise ,Klinische Forschergruppen“ einen geringeren Zugang zum Einsatz von Social Software haben als bspw. Projekt im Programm „Literaturversorgungs- und Informationssysteme“. Dies spiegelt sich auch in der Rücklaufquote der Antworten wieder (siehe Tabelle 1).

Tabelle 1: Personenanzahl je DFG-Programm für die Erhebung

\begin{tabular}{|c|c|c|c|}
\hline DFG-Programm & $\begin{array}{c}\text { A. } \\
\text { Befragte } \\
\text { Personen }\end{array}$ & $\begin{array}{c}\text { B. } \\
\text { Grundgesamtheit } \\
\text { an Personen }\end{array}$ & $\begin{array}{c}\text { C. } \\
\text { Antworten von } \\
\text { Projektleitern }\end{array}$ \\
\hline Literaturversorgung und Informationssysteme & 103 & 105 & $\begin{array}{c}14 \\
(14 \% \text { von } \mathrm{A}) \\
\end{array}$ \\
\hline Sonderforschungsbereiche & 94 & 498 & $\begin{array}{c}21 \\
(22 \% \text { von } A)\end{array}$ \\
\hline DFG-Forschungszentrum & 25 & 30 & $\begin{array}{c}1 \\
(4 \% \operatorname{von} A)\end{array}$ \\
\hline Forschergruppen & $\begin{array}{c}301 \\
(10 \% \text { von } B)\end{array}$ & $3.046^{1}$ & $\begin{array}{c}15 \\
(5 \% \text { von } A)\end{array}$ \\
\hline Klinische Forschergruppen & $\begin{array}{c}61 \\
(10 \% \text { von } B)\end{array}$ & 604 & $\begin{array}{c}3 \\
(0,5 \text { von } A)\end{array}$ \\
\hline Keine Angabe & & & 27 \\
\hline Insgesamt & 584 & 4.283 & $\begin{array}{c}81 \\
(13,84 \% \text { von } A)\end{array}$ \\
\hline
\end{tabular}

\subsection{Ergebnisse: Social Software in Forschungsprojekten der Deutschen Forschungsgemeinschaft}

\section{Bekanntheit und Nutrung von Social Software}

Die durchgeführte Befragung hat ergeben, dass 32,1\% der befragten Projektleiter in ihrer täglichen Arbeit, außerhalb ihrer Projekte, Social Software nutzen. 63\% setzen in ihrer täglichen Arbeit Wikis, 27\% Foren und 12\% Soziale Netzwerke und ebenfalls 12\% Instant Messenger ein. Weiterhin wurde von 7\% der Befragten geantwortet, dass sie in der Arbeitszeit auch auf andere Anwendungen wie Moodle, Google docs und Bug Tracking-System zurückgreifen, die in der Umfrage nicht explizit erfragt wurden (Antwort: Sonstiges).

Social Software wurde nur von ca. zwei Drittel der DFG-Verbundprojekten eingesetzt. Die Häufigkeit des Einsatzes verschiedener Tools in den Projekten und in Unternehmen ist weitestgehend identisch. Am häufigsten werden Wikis (43,2\%) eingesetzt. Foren werden am zweithäufigsten mit 12,3\% und Blogs zu 7,4\% (Tabelle 2) eingesetzt. Andere Web 2.0-Technologien werden sowohl in DFG-Forschungsprojekten als auch in Unternehmen finden nur in geringem Ausmaße Berücksichtigung. Von 81 befragten Projektleitern gaben $43(53,1 \%)$ an, Social Software in ihren kooperativen Forschungsprojekten einzusetzen. Den 43 Personen, die den Einsatz von Social Software bejahten, wurden ergänzend Fragen zur konkreten Handhabung der Social Software gestellt. Aus diesem Grund beziehen sich die nachfolgenden Auswertungen auf entweder die 43 Personen/Projekte, bei denen Social Software konkret eingesetzt wird bzw. auf alle befragten Personen $(\mathrm{n}=81)$.

Tabelle 2 zeigt, dass Social Software in der täglichen Arbeit oder für den privaten Gebrauch intensiver eingesetzt wird als in Projekten. Hieraus lässt sich schließen, dass Verbundprojekte nicht Treiber sondern

\footnotetext{
1 Im Rahmen der für diese Studie analysierten DFG-Programme für ,Forschergruppen' sind 3046 Personen in die insgesamt 3283 Projekte involviert. Um dieses DFG-Verfahren in unsere Untersuchung angemessen zu repräsentieren, haben wir $10 \%$ der involvierten Personen angeschrieben, die mittels einer einfachen Zufallsauswahl selektiert wurden.
} 
eher Nachzügler des Trends zum Einsatz von Social Software sind. Der Gebrauch von Social Software für projektunabhängige Arbeiten scheint sich demnach nicht auf den Einsatz in Forschungsprojekten niederzuschlagen.

Tabelle 2: Übersicht der Ergebnisse hinsichtlich der Nutzung von Social Software

\begin{tabular}{|c|c|c|c|}
\hline \multicolumn{2}{|l|}{ Quelle } & $\begin{array}{l}\text { Eigene } \\
\text { Analyse } \\
(2009)\end{array}$ & $\begin{array}{l}\text { Eigene } \\
\text { Analyse } \\
(2009)\end{array}$ \\
\hline \multicolumn{2}{|l|}{ Zielgruppe } & $\begin{array}{c}\text { projektunabhängiger } \\
\text { Einsatz } \\
n=81\end{array}$ & $\begin{array}{c}\text { Einsatz in DFG Verbund- } \\
\text { projekten } \\
n=81\end{array}$ \\
\hline \multicolumn{2}{|c|}{ Einsatz von Social Software } & $66,7 \%(53)$ & $53,1 \%(43)$ \\
\hline $\begin{array}{l}\text { Nutzung ver- } \\
\text { schiedener } \\
\text { Web 2.0- } \\
\text { Technologien } \\
(\mathrm{N}=131)\end{array}$ & $\begin{array}{l}\text { Wikis } \\
\text { Blogs } \\
\text { Foren } \\
\text { Soz. Netzwerke } \\
\text { Social Bookmarking } \\
\text { Instant Messaging } \\
\text { Weitere }\end{array}$ & $\begin{array}{c}63 \% \\
9,9 \% \\
27,2 \% \\
12,3 \% \\
3,7 \% \\
12,3 \% \\
7,2 \%\end{array}$ & $\begin{array}{c}43,2 \% \\
7,4 \% \\
12,3 \% \\
4,9 \% \\
0 \% \\
8,6 \% \\
14,8 \%\end{array}$ \\
\hline
\end{tabular}

Tabelle 3 zeigt, dass in 67\% aller Fälle lediglich ein Typ von Social Software in Projekten eingesetzt wird. Diese stellen in der Mehrzahl Blogs dar (Tabelle 2). In 23\% der Fälle kommen zwei Typen von Social Software zum Einsatz, in der Regel sind dies Wikis und Foren. In weniger als 10\% der Fälle werden mehr als zwei verschiedene Arten von Social Software in den Projekten eingesetzt (Tabelle 3). Der Einsatz von mehreren Social Software Technologien in einem Projekt könnte darauf zurückzuführen sein, dass diese Projekte über einen höheren Kooperationsbedarf oder ein höheres Kommunikationsbewusstsein verfügen.

Tabelle 3: Anzahl von Typen von Social Software in der Projektarbeit

\begin{tabular}{|l|r|r|r|r|}
\hline$n=43$ & Häufigkeit & Prozent & \multicolumn{1}{c|}{ Kumulierte Prozente } \\
\hline \multirow{3}{*}{$\begin{array}{l}\text { Anzahl eingesetzter Social Software Werk- } \\
z e u g e\end{array}$} & 1 & 29 & 67,4 & 67,4 \\
\cline { 2 - 5 } & 2 & 10 & 23,3 & 90,7 \\
\cline { 2 - 5 } & 4 & 4 & 9,3 & 100,0 \\
\cline { 2 - 5 } & & & & \\
\cline { 2 - 5 } & Gesamt & 43 & 100,0 & \\
\hline
\end{tabular}

Die Daten in Tabelle 4 verdeutlichen einen Zusammenhang zwischen der Intensität der Nutzung von Social Software im Rahmen des jeweiligen Projekts und in den projektunabhängigen Tätigkeiten des Projektleiters. Von denjenigen Projektleitern, die bereits außerhalb ihres Projektes Erfahrungen mit Social Software gesammelt haben, nutzen etwa 41\% mindestens einen Typ von Social Software auch in ihren Forschungsprojekten.

Tabelle 4: Einsatz von Social Software während und außerhalb der Projektarbeit

\begin{tabular}{|c|c|c|c|c|c|}
\hline \multirow[t]{2}{*}{$n=81$} & & & \multicolumn{2}{|c|}{$\begin{array}{c}\text { Einsatz von Social Software } \\
\text { im Projekt }\end{array}$} & \multirow[b]{2}{*}{ Gesamt } \\
\hline & & & Kein Einsatz & $\geq 1$ & \\
\hline \multirow{4}{*}{$\begin{array}{l}\text { Einsatz von Social } \\
\text { Software außerhalb } \\
\text { des Projektes }\end{array}$} & \multirow[t]{2}{*}{ Kein Einsatz } & Anzahl & 25 & 2 & 27 \\
\hline & & $\%$ der Gesamtzahl & $30,9 \%$ & $2,5 \%$ & $33,3 \%$ \\
\hline & \multirow[t]{2}{*}{$\geq 1$} & Anzahl & 13 & 41 & 54 \\
\hline & & $\%$ der Gesamtzahl & $16,0 \%$ & $50,6 \%$ & $66,7 \%$ \\
\hline \multirow{2}{*}{\multicolumn{2}{|c|}{ Gesamt }} & Anzahl & 38 & 43 & 81 \\
\hline & & $\%$ der Gesamtzahl & $46,9 \%$ & $53,1 \%$ & $100,0 \%$ \\
\hline
\end{tabular}

Tabelle 5 stellt den Einsatz von Social Software in den einzelnen für die Analyse herangezogenen Programmen der DFG dar. Besonders oft wird Social Software demnach in dem Programm „Literaturversorgungs- 
und Informationssysteme“ eingesetzt: von 14 Projekten nutzen 10 Projekte Social Software (72\%). Da diese Projekte per Definition IT-nah sind, verdeutlicht dies, dass IT-nahe Projekte auch eher auf den Einsatz neuer Technologien wie Social Software für die Projektorganisation zurückgreifen. Hier kann angenommen werden, dass Mitarbeiter in diesen Projekten bereits Kenntnisse im Umgang mit Social Software besitzen. Dieselbe Begründung kann ebenfalls für die niedrige Rücklaufquote der Befragung in dem Bereich „klinische Forschergruppen“ $(n=3)$ herangezogen werden. Die drei Projektleiter, die an der Umfrage teilgenommen haben, gaben ebenfalls an, keine Social Software in der Projektarbeit zu nutzen.

Tabelle 5: Social Software in unterschiedlichen DFG-Programmen

\begin{tabular}{|c|c|c|c|c|c|c|c|c|}
\hline \multirow[t]{2}{*}{$n=81$} & & \multicolumn{6}{|c|}{ DFG Programme } & \multirow[b]{2}{*}{$\begin{array}{l}\text { Ge- } \\
\text { samt }\end{array}$} \\
\hline & & $\begin{array}{l}\text { Keine } \\
\text { Angabe }\end{array}$ & $\begin{array}{l}\text { Literatur- } \\
\text { versor- } \\
\text { gungs- \& } \\
\text { Informa- } \\
\text { tions- } \\
\text { systeme }\end{array}$ & $\begin{array}{l}\text { DFG- } \\
\text { For- } \\
\text { schungs- } \\
\text { zentrum }\end{array}$ & $\begin{array}{l}\text { Sonderfor- } \\
\text { schungs- } \\
\text { bereich }\end{array}$ & $\begin{array}{l}\text { For- } \\
\text { schungs- } \\
\text { gruppen }\end{array}$ & $\begin{array}{l}\text { Klinische } \\
\text { Forscher- } \\
\text { gruppen }\end{array}$ & \\
\hline \multirow{2}{*}{$\begin{array}{l}\text { Einsatz von Social } \\
\text { Software im } \\
\text { Projekt }\end{array}$} & $\geq 1$ & 13 & 10 & 1 & 11 & 8 & 0 & 43 \\
\hline & $\overline{\text { Kein Einsatz }}$ & 14 & 4 & 0 & 10 & 7 & 3 & 38 \\
\hline \multicolumn{2}{|c|}{$\begin{array}{l}\text { Prozentualer Anteil - } \\
\text { Einsatz von Social Software }\end{array}$} & $48 \%$ & $71,5 \%$ & $100 \%$ & $52 \%$ & $53 \%$ & $0 \%$ & $53 \%$ \\
\hline \multicolumn{2}{|l|}{ Gesamt } & 27 & 14 & 1 & 21 & 15 & 3 & 81 \\
\hline
\end{tabular}

\section{Gründe für die Nicht-Nutzung und Bedenken im Einsatz, von Social Software}

Die größten Bedenken im Einsatz von Social Software haben die Befragten im wahrgenommenen zusätzlichen Aufwand der Nutzung und der Pflege der Inhalte (45,7\%). Überdies ist in dieser durchgeführten Studie für 34,6\% der Projektleiter der Nutzen von Web 2.0-Technologien unklar (Abbildung 1).

Hieraus kann abgeleitet werden, dass nicht nur das Nutzungsverhalten sondern auch die Einstellung zur Nutzung von Social Software in DFG-Forschungsprojekten eher dem Verhalten in KMU's und nicht dem in Großunternehmen ähnelt.

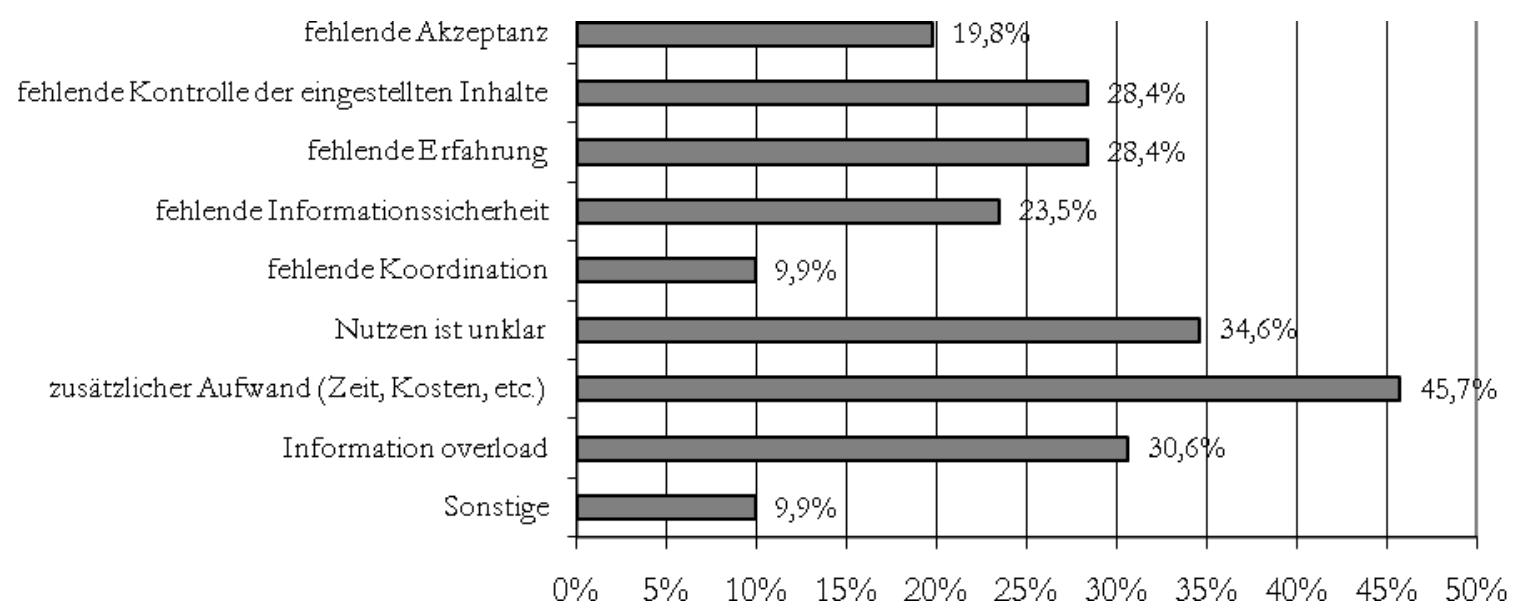

Abbildung 1: Bedenken zum Einsatz von Social Software ( $n=81$, Mehrfachnennungen möglich)

\section{Zufriedenheit mit Social Software}

Insgesamt sind 62\% der befragten Projektleiter sehr bis eher zufrieden mit dem Einsatz von Social Software und haben somit positive Erfahrungen im Einsatz in Projekten sammeln können. 


\section{Ziele und Mehrwerte im Einsatz mit Social Software}

In Unternehmen wird Social Software vor allem in den Bereichen Marketing und Vertrieb sowie im Kundendienst, zum Prosuming und zur Co-Creation eingesetzt (Lattemann et al. 2009). Die Generierung von User Generated Content bietet für über 50\% der Firmen zentrale Mehrwerte: Ziele der Kundenbindung, bspw. durch den Aufbau von Brand Communities, stellen darüber hinaus für Unternehmen vielversprechende Einsatzfelder für Social Software dar. Da DFG-Verbundprojekte im Gegensatz zu Unternehmen keine Kundenorientierung aufweisen, liegt der Fokus hier naturgemäß auf internen Koordinationszielen und nicht auf der Kommunikation mit Kunden. Die Zusammenarbeit des Projektteams (34,6\%), Wissensmanagement (32,1\%), Unterstützung von kollaborativen Arbeiten (30,9\%) und Informationsmanagement (25,9\%) werden in DFGVerbundprojekten als wesentliche Ziele des Einsatzes von Social Software genannt (Abbildung 2).

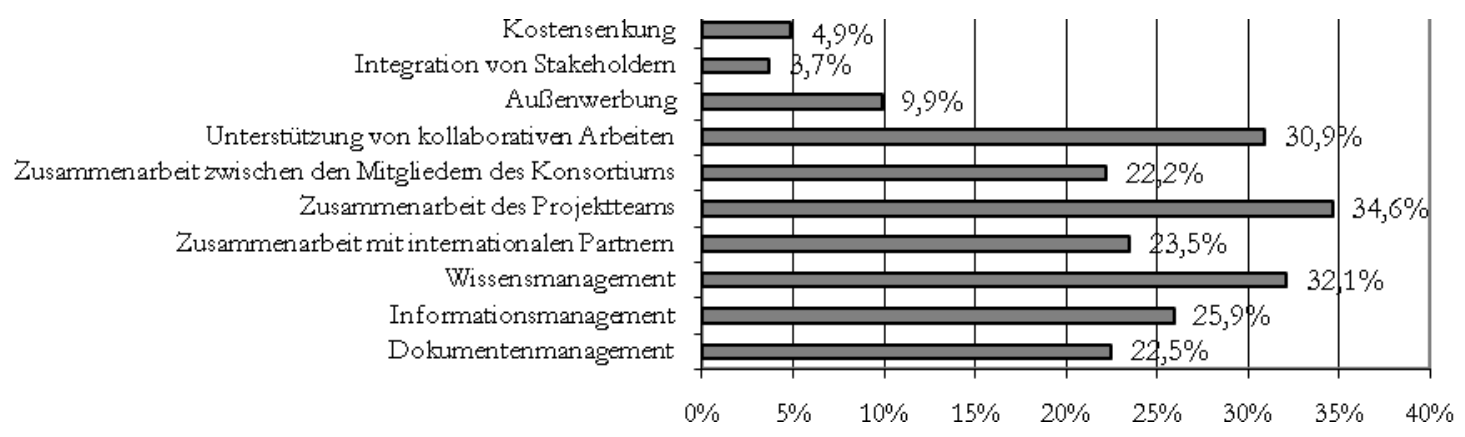

Abbildung 2: Ziele des Einsatzes von Social Software (n=81, Mehrfachnennungen möglich)

$42 \%$ der befragten Projektleiter, die Social Software nutzen, geben an, dass durch den Einsatz von Social Software eine verbesserte Zusammenarbeit ermöglicht wird. Weitere Vorteile liegen in der effizienten Nutzung von Wissen $(35,8 \%)$, in der vereinfachten Koordination $(34,6 \%)$ und in der verbesserten Kommunikation $(33,3 \%)$ (Abbildung 3). Durch den Einsatz von Social Software kann demnach im Projekt vorhandenes Wissen an einem für jeden Mitarbeiter schnell zugänglichen Ort gesichert und allen zur Verfügung gestellt werden. Hierdurch kann ein gemeinsamer Wissensstand gepflegt und ausgebaut werden.

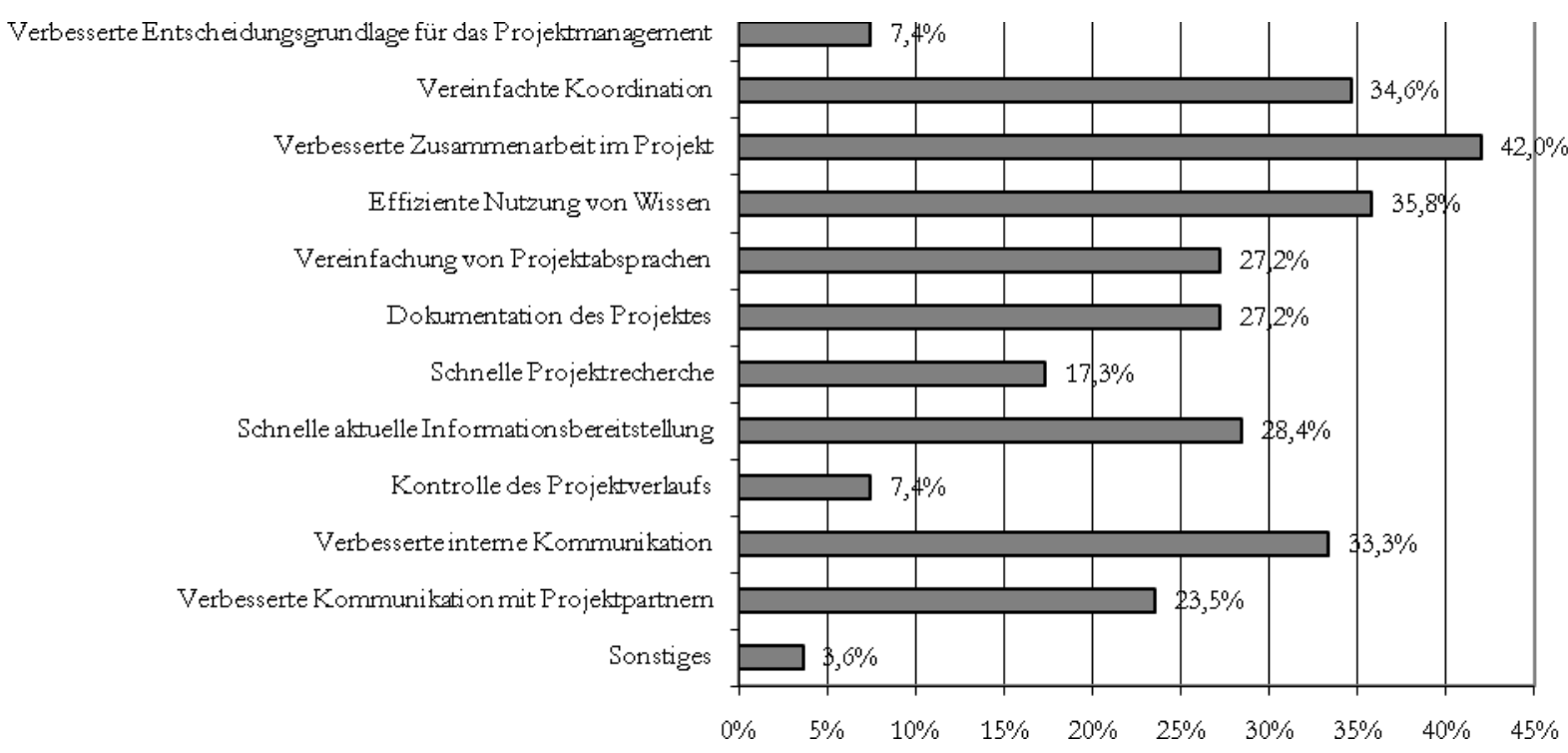

Abbildung 3: Vorteile des Einsatzes von Social Software ( $n=81$, Mehrfachnennungen möglich)

\section{Ansiedlung der externen Projektpartner}

Von den 81 befragten Projektleitern mit vollständig ausgefüllten Fragebogen gaben 66 an, dass sie mit externen, unabhängigen Projektpartnern außerhalb ihrer Heimatinstitution im Projekt zusammenarbeiten. 35 Projekte haben zwei und mehr externe Partner. In 88,4\% der Projekte, in denen Social Software eingesetzt wird, sind zwei oder mehr unabhängige Projektpartner involviert. Es zeichnet sich die Tendenz ab, dass bei größe- 
rer Anzahl unabhängiger Partner im Projekt die Intensität im Einsatz von Social Software in Projekten zunimmt (Tabelle 6). Kooperative Forschungsprojekte setzen Social Software insbesondere bei geografisch verteilten Teams ein (vergleiche Tabelle 8).

Tabelle 6: Social Software in Forschungsprojekten in Relation zur Anzahl der Partner

\begin{tabular}{|c|c|c|c|c|c|c|c|c|c|}
\hline \multirow{2}{*}{\multicolumn{2}{|c|}{$n=81$}} & \multicolumn{7}{|c|}{ Anzahl unabhängiger Partner im Projekt } & \multirow{2}{*}{$\begin{array}{l}\text { Ge- } \\
\text { samt }\end{array}$} \\
\hline & & - & 2 & 3 & 4 & 5 & 6 & 7 & \\
\hline \multirow{2}{*}{$\begin{array}{l}\text { Software Ein- } \\
\text { satz im Projekt }\end{array}$} & $\geq 1$ & 5 & 21 & 9 & 4 & 2 & 1 & 1 & 43 \\
\hline & Kein Einsatz & 10 & 10 & 13 & 3 & 1 & 0 & 1 & 38 \\
\hline \multicolumn{2}{|l|}{ Anteil } & $33 \%$ & $67 \%$ & $41 \%$ & $57 \%$ & $67 \%$ & $100 \%$ & $50 \%$ & $53 \%$ \\
\hline \multicolumn{2}{|l|}{ Gesamt } & 15 & 31 & 22 & 7 & 3 & 1 & 2 & 81 \\
\hline
\end{tabular}

Ebenso wurde untersucht, inwiefern ein Zusammenhang zwischen der Teamgröße eines Forschungsprojektes und Nutzung von Social Software besteht (Tabelle 7). Hier zeigt sich, dass insbesondere Projekte mit 6-10 Personen (30,2\%), Projekte bis 5 Personen $(27,9 \%)$ und Projekt mit über 30 Personen (20,9\%) besonders häufig Social Software einsetzen. Ein Zusammenhang zwischen der Anzahl der Projektmitarbeiter und dem Einsatz von Social Software ist jedoch nicht eindeutig erkennbar, da kleine Teams mit weniger als 5 Mitarbeitern ebenfalls zu etwa $28 \%$ Social Software einsetzen. Hieraus lässt sich schließen, dass der Einsatz von Social Software für jede Teamgröße Vorteile bringen kann. Ausschlaggebend für den Einsatz von Social Software sind vielmehr die Verortung der einzelnen Projektpartner (vergleiche Tabelle 8).

Tabelle 7: Social Software in Forschungsprojekten in Relation zur Teamgröße

\begin{tabular}{|c|c|c|c|c|c|c|c|c|c|c|}
\hline \multicolumn{3}{|l|}{$n=43$} & \multicolumn{7}{|c|}{ Anzahl Personen im Projekt } & \multirow{2}{*}{$\begin{array}{l}\text { Ge- } \\
\text { samt }\end{array}$} \\
\hline & & & $\leq 5$ & $6-10$ & $11-15$ & $16-20$ & $21-25$ & $26-30$ & $>30$ & \\
\hline \multirow{2}{*}{$\begin{array}{l}\text { Einsatz von } \\
\text { Social Soft- } \\
\text { ware im Pro- } \\
\text { jekt }\end{array}$} & \multirow{2}{*}{$\begin{array}{l}\text { es wird min- } \\
\text { destens eine } \\
\text { Software } \\
\text { eingesetzt }\end{array}$} & Anzahl & 12 & 13 & 4 & 2 & 2 & 1 & 9 & 43 \\
\hline & & Anteil & $27,9 \%$ & $30,2 \%$ & $9,3 \%$ & $4,7 \%$ & $4,7 \%$ & $2,3 \%$ & $20,9 \%$ & $100 \%$ \\
\hline
\end{tabular}

Ein eindeutiger linearer Zusammenhang zwischen der Entfernung der Projektpartner und der Intensität des Einsatzes von Social Software für die Projektarbeit konnte ebenso nicht nachgewiesen werden. Jedoch zeigt sich, dass Projekte, bei denen die Projektpartner in unmittelbarer Nähe verortet sind, signifikant weniger Social Software in den Projekten einsetzen als in lokal oder international ausgerichteten Projekten (Tabelle 8). Eine Begründung hierfür könnte sein, dass sich die Teams innerhalb eines Gebäudes befinden und eine direkte Kommunikation der computerbasierten Zusammenarbeit vorziehen.

Tabelle 8: Social Software in Projekten in Relation zur räumlichen Verteilung

\begin{tabular}{|c|c|c|c|c|c|c|c|c|c|}
\hline \multicolumn{2}{|c|}{} & \multicolumn{9}{|c|}{ Räumliche Verteilung der Partner } & & \\
\cline { 3 - 13 } & & $\begin{array}{c}\text { Keine } \\
\text { Anga- } \\
\text { ben }\end{array}$ & $\begin{array}{c}\text { in un- } \\
\text { mittel- } \\
\text { barer } \\
\text { Nähe }\end{array}$ & lokal & regional & $\begin{array}{c}\text { über- } \\
\text { regional }\end{array}$ & national & $\begin{array}{c}\text { inter- } \\
\text { national }\end{array}$ & Gesamt \\
\hline $\begin{array}{c}\text { Software } \\
\begin{array}{c}\text { Einsatz im } \\
\text { Projekt }\end{array}\end{array}$ & Anzahl & 6 & 10 & 10 & 7 & 8 & 19 & 11 & 81 \\
\hline & Anteil & - & 2 & 7 & 4 & 6 & 10 & 8 & 37 \\
\hline
\end{tabular}

40 Projektleiter, die bereits in ihren Forschungsprojekten Social Software einsetzen, werden dies auch in Zukunft tun (49,3\%). Von denen, die bisher keine Social Software einsetzen, beabsichtigen 22,2\% in Zukunft diese Software einzusetzen (Tabelle 9). Dieser Trend der zunehmenden Bedeutung und des Einsatzes von Social Software in Projekten deckt sich mit Studienergebnissen zum Einsatz von Social Software in Unternehmen (BITKOM 2008; Berlecon Research 2007). Die praktische Bedeutung von Web 2.0 wird demnach in Zukunft deutlich zunehmen. 
Tabelle 9: Zukünftiger Einsatz von Social Software in DFG-Verbundprojekten

\begin{tabular}{|l|l|c|c|c|}
\hline \multirow{2}{*}{\begin{tabular}{l} 
n=81 \\
\cline { 3 - 5 }
\end{tabular}} & \multicolumn{2}{|c|}{ Einsatz in Zukunft } & \multirow{2}{*}{ Gesamt } \\
\cline { 3 - 5 } $\begin{array}{l}\text { Derzeitiger Soft- } \\
\text { ware Einsatz }\end{array}$ & Kein Einsatz & $20(25 \%)$ & $18(22 \%)$ & 38 \\
\cline { 2 - 5 } & $>1$ Social Software Typ & $3(4 \%)$ & $40(49 \%)$ & 43 \\
\hline Gesamt & $23(28 \%)$ & $58(71 \%)$ & 81 \\
\hline
\end{tabular}

\section{$4 \quad$ Fazit und Ausblick}

Social Software kann Projekte primär hinsichtlich zweier Aspekte unterstützen. Erstens in der Dokumentation von Projektergebnissen und Datensammlung über Foren und zum zweiten in der kollaborativen Arbeit über Wikis (Koch 2008a). Die Ergebnisse zeigen, dass auch primär diese beiden Werkzeuge in Projekten eingesetzt werden. Die dargestellten Untersuchungsergebnisse zeigen, dass der Nutzen und der Mehrwert von Social Software in Forschungsverbundprojekten ebenso wie in der Wirtschaft erkannt werden. Wurde Social Software in Projekten eingesetzt, waren die Nutzer überwiegende „,sehr zufrieden“ oder „eher zufrieden“. Die Intensität der Nutzung von Social Software in Forschungsverbundprojekten ist jedoch lediglich vergleichbar mit der Nutzung dieser Technologien in KMUs und liegt damit deutlich hinter der Nutzung in Großunternehmen und in Unternehmen aus der Informationswirtschaft in Deutschland. Die Untersuchungsergebnisse weisen darauf hin, dass der Nutzen von Social Software für die verteilte Projektarbeit klärungsbedürftig ist. Auch scheinen die derzeitigen Modi zur Einführung von Social Software in die Projektarbeit nicht ausreichend. In knapp 29,6\% der Projekte wird das Wissen für den Umgang mit der Technologie vorausgesetzt und nicht explizit erklärt. 25,9\% sagen, dass die Bedienung der Technologie selbstständig erarbeitet werden muss. Anders als in Unternehmen, die in der Regel ausreichend Ressourcen investieren können, um Web 2.0-Plattformen aufzusetzen und das notwendige Know-How aufzubauen, sind die Finanzmittel und insbesondere der Zeitrahmen in Forschungsprojekten beschränkt, die für solche Instrumente aufgebracht werden können. Gleichzeitig ist das im Forscherteam zur Verfügung stehende Wissen stark von der Ausrichtung des Projekts geprägt (bspw. IT-Projekte).

Dies hemmt die verbreitete Nutzung dieser Technologien und Konzepte. Hier besteht also Aufholbedarf in der Erklärung zum Einsatz. Um diese Lücke zu schließen und um die Potenziale auch für ihre Projekte auszunutzen, könnte die DFG pro-aktive die Nutzung von Social Software empfehlen oder Partner aus der Wirtschaft bewusst in die Projekte integrieren, so dass Erfahrungen mit der Nutzung der Social Software aktiv ausgetauscht werden können. 29,3\% der Befragten befürworten, dass der Projektträger Social Software bereitstellen soll, damit diese Werkzeuge verstärkt im Projekt eingesetzt werden. Hierzu ist zunächst die Entwicklung von Instrumenten notwendig, die speziell darauf ausgerichtet sind, die in Forschungsprojekten herrschenden Bedürfnisse zur erfüllen. So hat die Untersuchung gezeigt, dass eine leichte Bedienbarkeit und unproblematische Implementierung hierfür notwendige Kriterien darstellen.

Die vorgestellten Ergebnisse sind jedoch mit Vorsicht zu interpretieren. Zum einen besteht ein möglicher Bias in den Daten, da durch die ausschließliche Befragung über einen Online-Fragebogen angenommen werden kann, dass sich verstärkt internetaffine Projektleiter beteiligt haben und somit die Daten positiver in Richtung der Nutzung sozialer Software verschoben sind. Weiterhin ist die untersuchte Fallzahl mit 81 gering. Für einige Auswertungen musste sogar auf eine noch kleinere Untersuchungsgruppe zurück gegriffen werden. Eine Verallgemeinerung oder Übertragung der erhaltenen Daten auf andere Bereiche ist daher nur sehr eingeschränkt möglich. Auch ist eine recht starke Variabilität der Ergebnisse zwischen Fachgebieten und Projektarten zu erkennen. Naturgemäß findet ein intensiverer Einsatz insbesondere in Projekten statt, die informationstechnische Themen behandeln. So bestätigen alle 14 Projektleiter aus dem Bereich Literaturversorgungs- und Informationssysteme den Bedarf an Social Software in der Projektarbeit, zehn von Ihnen setzen Social Software ein.

Diese Untersuchung zum Einsatz von Social Software in kooperativen Forschungsprojekten hatte zum Ziel, Projektleitern den aktuellen Status Quo zu vermitteln. Um ein umfassendes Bild zu erhalten und da im Vorfeld nur wenige Informationen über das Forschungsobjekt vorlagen, wurde als Methodik ein exploratives Vorgehen gewählt. Mittels der durchgeführten Befragung wurde eine Grundlage für folgende und angrenzende Forschungsvorhaben und -methoden wie Usability Experimente oder Expertenbefragungen geschaffen. Ergänzend könnte ein Strukturmodell Aufschluss darüber geben, in was für einem Abhängigkeitsverhältnis 
Variablen zueinander stehen, um daraus allgemeingültige Erfolgsfaktoren für den Einsatz von Social Software abzuleiten.

Weitere Studien, die den Einsatz von Social Software in Industrie- und Forschungsprojekten gegenüberstellen, sollten in Zukunft auch im internationalen Vergleich, durchgeführt werden. Hierdurch können Erkenntnisse zum aktuellen Status quo deutscher Projekte im europäischen und globalen Vergleich erlangt werden.

\section{Literatur}

Allen C (2004) Tracing the evolution of social software. http://www.lifewithalacrity.com/2004/10/tracing_the_evo.html, Zugriff am 28.09.2009.

Bächle M (2006) Social Software. INFORMATIK SPEKTRUM 29(2):121-124.

Bartel T (2006) Wikis als Wissensmanagement unterstützende Systeme in Unternehmen. Diplomarbeit, Universität zu Köln.

Berlecon Research (2007) Berlecon Research: Enterprise 2.0 in Deutschland. http://www.whoiswho.de/CoreMedia_Berlecon_Enterprise2.0.pdf, Zugriff am 12.12.2008.

BITKOM e.V. (2008) BITKOM: Enterprise 2.0. Analyse zu Stand und Perspektiven in der deutschen Wirtschaft, 2008. www.bitkom.org/files/documents/BITKOM-Studie_Enterprise_2Punkt0.pdf, Zugriff am 12.12.2008.

Chesbrough HW (2003) Open innovation. The new imperative for creating and profiting from technology. Harvard Business School Press, Boston.

Coates, T (2005) http://www.plasticbag.org/archives/2005/01/ an_addendum_to_a_definition_of_social_software/,Zugriff am 16.9.2009.

Döbler T (01/2007) Marktchancen durch Social Software. FAZIT-Studie Stiftung Baden-Württemberg.

Economist Intelligence Unit (2007) The Economist: Serious business web 2.0 goes corporate - A report from the economist intelligence unit Sponsored by FAST, 2007. http://graphics.eiu.com/upload/eb/fast_report.pdf, Zugriff am 12.12.2008.

Economist Intelligence Unit (2008) The Economist: The digital company 2013 - How technology will empower the customer, 2008.

http://www.nokiaforbusiness.com/Page\%20Content/Mobilize\%20your\%20business/Knowledge\%20ce nter/White\%20Papers/Whitepaper_digital2013.pdf, Zugriff am 12.12.2008.

GPM 2009 http://www.gpm-ipma.de//docs/showsite.php?menu=0102040123, Zugriff am 28.09.2009.

Koch M (2008a) Lehre aus der Vergangenheit - Computer-Supported Collaborative Work \& Co. Buhse W/Stamer S (2008) Enterprise 2.0: Die Kunst, Loszulassen. Rhombos:37-58.

Koch M (2008b) Enterprise 2.0...Social Software in Unternehmen. Whitepaper, Bundeswehr Universität München.

Koch M, Richter A (2007) Enterprise 2.0: Planung, Einführung und erfolgreicher Einsatz von Social Software in Unternehmen. Oldenbourg Wissenschaftsverlag, München.

Krause S (2009) Geleitwort Enterprise 2.0: Planung, Einführung und erfolgreicher Einsatz von Social Software in Unternehmen. Oldenbourg, München.

Lattemann C, Kupke S, Stieglitz S (2009) Deutsche Unternehmen auf dem Weg zum Web 2.0? - Eine Bestandsaufnahme, in: HMD - Praxis der Wirtschaftsinformatik, Special Issue „E-Collaboration“, Heft Nr. 267, S.18-26.

McAfee A (2009) Enterprise 2.0: New collaborative tools for your organization's toughest challenges. Harvard Business Press, Boston. 
McKinsey (2007) How businesses are using web 2.0: A McKinsey Global Study, 2007. http://www.mckinsey.de/downloads/publikation/mck_on_bt/2007/mobt_12_How_Businesses_are_U sing-Web_2_0.pdf, Zugriff am 12.12.2008.

McKinsey (2008) Building the web 2.0 enterprise: A McKinsey Global Study 2008, http://www.openinnovation.eu/download/Mckinsey\%20July\%202008.pdf, Zugriff am 12.12.2008.

O'Reilly T (2005) What is web 2.0 - Design patterns and business models for the next generation of software. http://www.oreillynet.com/lpt/a/6228.

Orth R (2009) Wissenmanagement mit Wiki-Systemen. Springer-Verlag, Heidelberg.

Piller F, Stotko C (2003) Mass Customization und Kunden-Integration: neue Wege zum innovativen Produkt. Symposion, Düsseldorf.

Scheer AW (2008) Dem Enterprise 2.0 gehört die Zukunft. Buhse, Willms/Reinhard, Ulrike (2008) DNADIGITAL - Wenn Anzugträger auf Kapuzenpullis treffen, DNAdigital_04 - Der Weg zum Enterprise 2.0.. http://www.scribd.com/doc/12544534/DNAdigital-Wenn-Kapuzenpullis-aufAnzugtraeger-treffen, Stand: 13.09.2009. dmp Digital- und Offsetdruck, Berlin:23-25.

Stieglitz S (2008) Steuerung Virtueller Communities: Instrumente, Mechanismen, Wirkungszusammenhänge. Gabler, Wiesbaden.

Stieglitz, S, Lattemann, C, vom Brocke, J, Sonnenberg, C (2008) Economics of virtual communities - A financial analysis of a case study at the Berlin stock exchange, in: Proceedings of the 21st Bled eCollaboration: Overcoming Boundaries through Multi-Channel Interaction, S. 248-262.

Stieglitz S, Schneider AM, Lattemann C (2008) The impact of social software on customer decision making processes. In: E-Business Review, 8. Jg.

Szugat M, Gewehr JE, Lochmann C (2006) Social Software. Entwickler.press, Imprint der Software \& Support Verlag GmbH.

Techconsult GmbH (2008) IT und E-Business im Mittelstand 2008, Studie im Auftrag von IBM und der Zeitschrift Impulse. http://www.impulse.de/downloads/impulse_IBM_Studie_2008.pdf, Zugriff am 12.12.2008. 